\title{
TIME-AND-SPACE-DOMAIN STUDY OF DIFFRACTING AND NON-DIFFRACTING LIGHT PULSES
}

\author{
P. Saari ${ }^{\text {a }}$, P. Bowlan ${ }^{\mathrm{b}}$, H. Valtna-Lukner ${ }^{\mathrm{a}}$, M. Lõhmus ${ }^{\mathrm{a}}$, P. Piksarv ${ }^{\mathrm{a}}$, and R. Trebino ${ }^{\mathrm{b}}$ \\ a Institute of Physics, University of Tartu, 142 Riia St, Tartu, 51014 Estonia \\ E-mail: peeter.saari@ut.ee \\ ${ }^{\mathrm{b}}$ School of Physics, Georgia Institute of Technology, 837 State St NW, Atlanta, GA 30332, USA
}

Received 27 October 2009; revised 17 February 2009; accepted 19 March 2010

\begin{abstract}
We present an overview of our very recent results on the evolution of ultrashort pulses after propagating through various optical elements. Direct spatiotemporal measurements of the electric field were made using the technique SEA TADPOLE. Our SEA TADPOLE device can resolve spatial features as small as $\sim 5 \mu \mathrm{m}$ and temporal features as small as $\sim 5$ fs. The experimental results are verified by theoretical calculations. The superluminality of pulses with Bessel-function-like radial profiles is discussed.
\end{abstract}

Keywords: Bessel beam, boundary diffraction wave, Bessel-X pulse, superluminal propagation, Arago spot

PACS: $42.25 . \mathrm{Fx}$, 42.25.Gy, 42.65.Re, 42.79.Bh

\section{Introduction}

In 1987 Bessel light beams [1] were introduced and now constitute a mature field with numerous applications (see review [2]). These beams are important because they possess a controversial quality: they are "diffraction-free" and so preserve their tightly focused central bright spot over large distances of propagation as if the beam were not obeying the laws of diffraction. At the same time, quite independently, in mathematical physics, the topic of undistorted or localized waves emerged, dealing with ultrabroadband pulses that are not only "diffraction-free" in space but also propagate without any spread in time [3-5]: "light bullets" or "electromagnetic missiles." To date, various localized waves propagating in vacuum superluminally (faster than the speed of light in vacuum), luminally, or subluminally have been studied in detail, and promising applications have been proposed (see, e. g., reviews [6-10] and the first monograph [11] on the field). The feasibility of such light bullets moving faster than $c$ has been experimentally demonstrated more than once [12-17], but, from time to time, papers still appear in which the superluminal group velocity in vacuum of such wave packets is questioned. Therefore, recently [18] we accomplished, for the first time, with appropriately high resolution and accuracy, a direct spatiotemporal measurement of the electric field and propagation velocity of the simplest superluminal localized wave the so-called Bessel-X pulse [13], which comprises an energy lump of a micrometre in diameter at the joint apex of a sparse double-conical wave. In this paper, we first present an overview of this result.

Secondly, we touch briefly on our spatiotemporal measurements of accelerating and decelerating Bessel pulses [19]. The term was proposed in [20] where the generation and properties of such pulses were theoretically investigated. These pulses are similar to the Bessel-X pulses, with the main difference being that they are generated by crossing and interfering focusing (or defocusing) pulses, which have curved pulse fronts and form part of a spindle torus surface, rather than the double conical surface of Bessel-X pulses. As a result, their bullet-like, central, intense apex and accompanying Bessel rings become smaller or larger as the pulse propagates, depending on whether the torus shrinks towards a ring or expands towards a sphere. But the central spot of these pulses is still localized and intense over a propagation distance considerably longer than that of a Gaussian beam with a comparable waist size.

The third topic that we will discuss involves viewing simple, well-known cases of diffraction, but in the time domain. The bending of light waves in the shadow region behind an opaque disk and the appearance of a bright "Spot of Arago" in the shadow centre are well-known manifestations of diffraction. Tremendous 
progress was made in the mathematical treatment of diffraction, resulting in the well developed theory with Fresnel-Kirchhoff and Rayleigh-Sommerfeld versions (see, e. g., monographs $[21,22]$ and references therein). An alternative theory, inspired by the early ideas of Thomas Young, has been developed by Maggi [23], Rubinowicz [24], Miyamoto, and Wolf (references given in [22]). The boundary diffraction wave (BDW) theory, as it was called, describes diffraction from openings in opaque screens in a mathematically simple manner. The BDW theory is especially intuitive when describing the formation of the diffracted field for the case of illumination with ultrashort laser pulses.

Contrary to the traditional treatment using monochromatic fields, in which the transmitted waves fill large depths of space behind the screen and overlap with each other there, ultrashort pulses - typically only few micrometres "thick" - behave almost like a solitary wave-front surface. Hence, the time-domain study of diffraction in terms of pulsed BDWs is not only didactically preferable but also opens new interesting directions and applications, such as in the study of focusing and other transformations of ultrashort pulses (see, e. g., paper [25] and references therein). The formation of an ultrashort boundary wave pulse just after a circular aperture has been theoretically studied [26], and experimental evidence for its existence was obtained by measuring modulations in the spectrum of the on-axis field as well as with CCD-recordings of the time-integrated radial intensity distribution of the field [27]. Our aim has been to directly record, with simultaneous spatial and temporal resolution, the evolution and interference of the boundary waves behind various screens. The results obtained are presented in the paper by Lõhmus et al. which can be found in this issue, and here we consider only the spot of Arago.

\section{Spatiotemporal measurement of light fields}

In our experiments we used a KM Labs Ti:Sa oscillator with $33 \mathrm{~nm}$ of bandwidth (FWHM) and an approximately Gaussian spectrum with a central wavelength $\lambda_{0}=805 \mathrm{~nm}$. The spot size of the laser beam was $4 \mathrm{~mm}$ (FWHM). Our measurements not only required high spatiotemporal resolution, but also high sensitivity. First of all, we routinely measure the relatively intense, spatially uniform pulse directly out of our laser, which is the input pulse in these experiments, and which also acts as a reference pulse in the measurements, using the FROG technique [28]. To obtain ultrahigh spatiotemporal resolution in both the intensity and phase, in conjunction with the required sensitivity, we used a technique called SEA TADPOLE (Spatially Encoded Arrangement for Temporal Analysis by Dispersing a Pair of Light E-fields [29]), which is based on spectral interferometry. It involves measuring the spectrum of the sum of the known reference pulse and the unknown pulse to yield the unknown pulse's temporal field. This approach is much like monochromatic-beam spatial interferometry or holography, where measurement of the spatial intensity of the sum of a known spatial field and an unknown monochromatic wave yields the unknown wave field in space. Finally, we achieve the high spatial resolution of the unknown field by simply scanning the micrometre-sized tip of the SEA TADPOLE input fibre point-by-point through the space where the unknown light field propagates. SEA TADPOLE has demonstrated a spatial resolution as small as 0.5 micrometres by using near-field scanning optical microscopy fibre tips, but 5 micrometres is sufficient for these measurements, allowing the use of standard off-the-shelf fibres. A description of the SEA TADPOLE set-up used can be found in Refs. $[18,25]$ and in the paper by Lõhmus et al. in the given issue. The plots from our SEA TADPOLE measurements, which are shown below, can be viewed as still images or "snapshots in flight," since they are spatiotemporal slices of the magnitude of the electric field $|E(x, y, z, t)|$ of the pulses.

\section{Results on "diffraction-free" Bessel-X pulse}

The most effective Bessel beam generator - a conical lens (axicon) - refracts plane waves towards its axis and thus shapes a femtosecond pulse into the Bessel-X pulse with its characteristic double-conical profile, as shown in Fig. 1. If the aperture radius $R$ of the axicon were infinitely large, the pulse would propagate rigidly and without any spread of its micrometre-size central bright spot at the joint apex of the cones over an infinitely large distance. In the case of a limited aperture, it follows from the geometry that the depth of the invariant propagation of the pulse (let us call it the Bessel zone) is restricted to $z_{\mathrm{B}}=R / \tan \theta$, where $\theta$ (the socalled axicon angle) is the angle of inclination of the wave vectors of the constituent plane waves toward the axis $z$.

Some measured "snapshots" of propagation of the Bessel-X pulse are shown in Fig. 1, together with theoretical simulations (this time calculated as an axisymmetric superposition of plane waves with a Gaussian aperture). The two are in good agreement except that the wings in the $z=5.5 \mathrm{~cm}$ image are shorter in the 

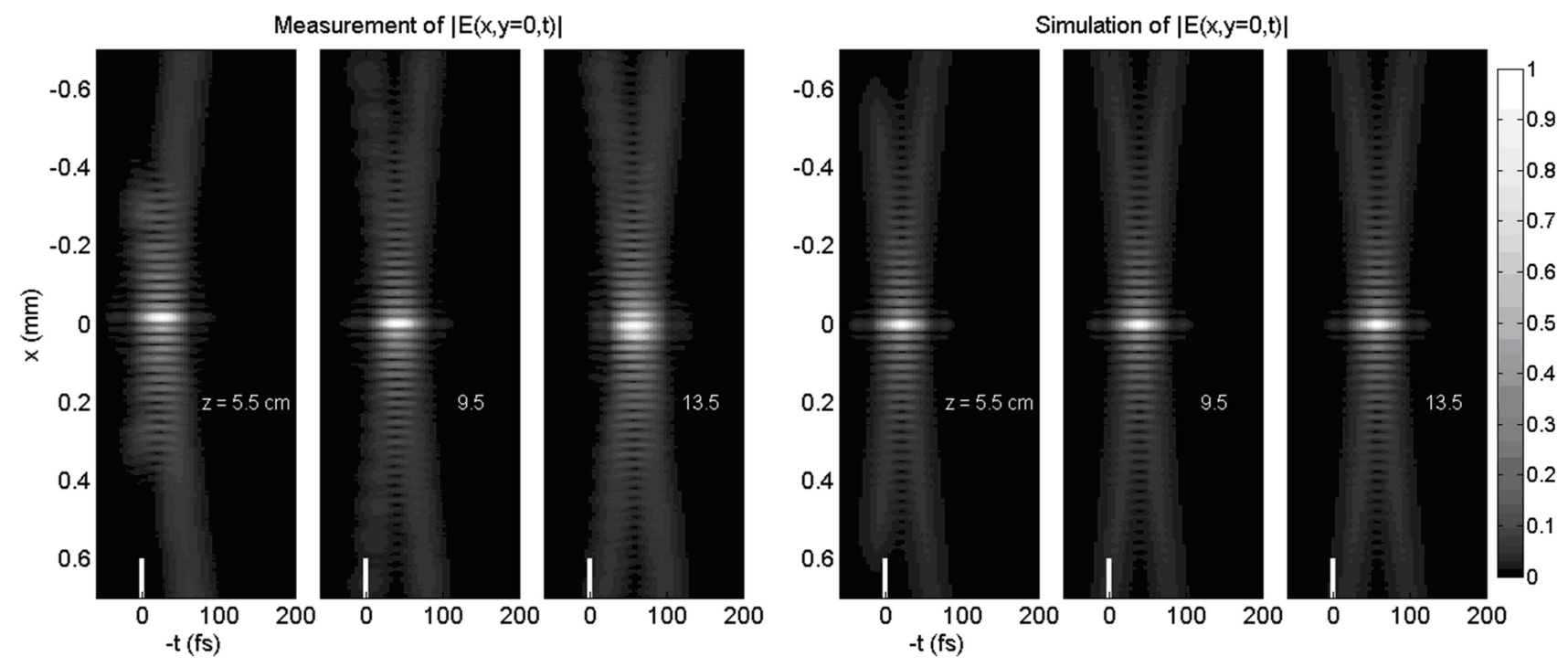

Fig. 1. Left: the measured field amplitude at three different distances $(z)$ after the axicon. Right: the corresponding simulations. The greyscale bar indicates the amplitude, and we have normalized each field to have a maximum of 1 . The white bar on the time axis emphasizes $t=0$ relative to the reference pulse, which is where the pulse would be located if it were propagating at velocity $c$. The "thickness" of each of the X-branches indicates the duration of the input (and reference) pulse.

measurement. This is because axicons are difficult to machine perfectly; in particular, the tip of the cone is always distorted, so the Bessel zone is shorter than what would be expected in the ideal case.

There are several interesting features in these plots. The central maximum of the pulse has a width of $\sim 20 \mu \mathrm{m}$, which - as well as the coaxial intensity rings surrounding it - remains essentially unchanged in shape from $z=5 \mathrm{~cm}$ through $z=13.5 \mathrm{~cm}$. Thus the apex flies rigidly as a light bullet together with its sparse wings at constant speed. This is because the Bessel-X pulse is a propagation-invariant conical wave. Also, the Bessel$\mathrm{X}$ pulse's superluminal speed is apparent in these plots. SEA TADPOLE measures the pulse's arrival time with respect to the reference pulse, which travels at the speed of light $(c)$. Therefore, if the Bessel-X pulse were travelling at the speed of light, then at each $z$ its spatiotemporal intensity would have the same centre on the time axis (here $t=0$ and emphasized with the white line), but it is easy to see that this is not the case. From the axicon angle value $\theta=0.92^{\circ}$ (corresponds to our axicon's apex angle $176^{\circ}$ ) as well as from the simulations, we find that the Bessel-X pulse's speed (axial group velocity) should be $1.00013 \mathrm{c}$. From our experimental plots we determined [18] it to be $1.00012 c$ - within $0.001 \%$ error of the expected value.

\section{Results on accelerating and decelerating Bessel pulses}

In order to generate accelerating (or decelerating) pulses we mounted a lens with focal length of $+153 \mathrm{~mm}$ (or $-152 \mathrm{~mm}$ ) before the axicon. All these results were published in [19], and here we restrict ourselves to the decelerating pulse case only.

The spatiotemporal profiles of the decelerating Bessel pulse at nine positions were measured. In all cases, we measured the complete spatiotemporal intensity and phase, but we show only the spatiotemporal intensities here, as this information is more interesting. Three of these measurements for each case are shown in Fig. 2. For comparison, numerical simulations were carried out, and as seen in the figure, the two are in very good agreement. The $\mathrm{X}$-branching corresponding to the double conical profile of the pulse is not seen because, due to the lens and deceleration, the axicon angle $\theta$ is less than in Fig. 1 and decreases with propagation distance. Due to the negative lens the fronts of the pulses (and phase fronts) obtain a curvature which decreases in the course of propagation and therefore the axicon angle $\theta$ on the axis also decreases - resulting in deceleration of the movement of the strong interference field on the axis, which is still in a good approximation nothing but a Bessel-X pulse.

SEA TADPOLE measures the pulse's arrival time with respect to the reference pulse, the latter of which, after passing through the compensating piece of glass, travels at the speed of light $c$. The origin of our time 

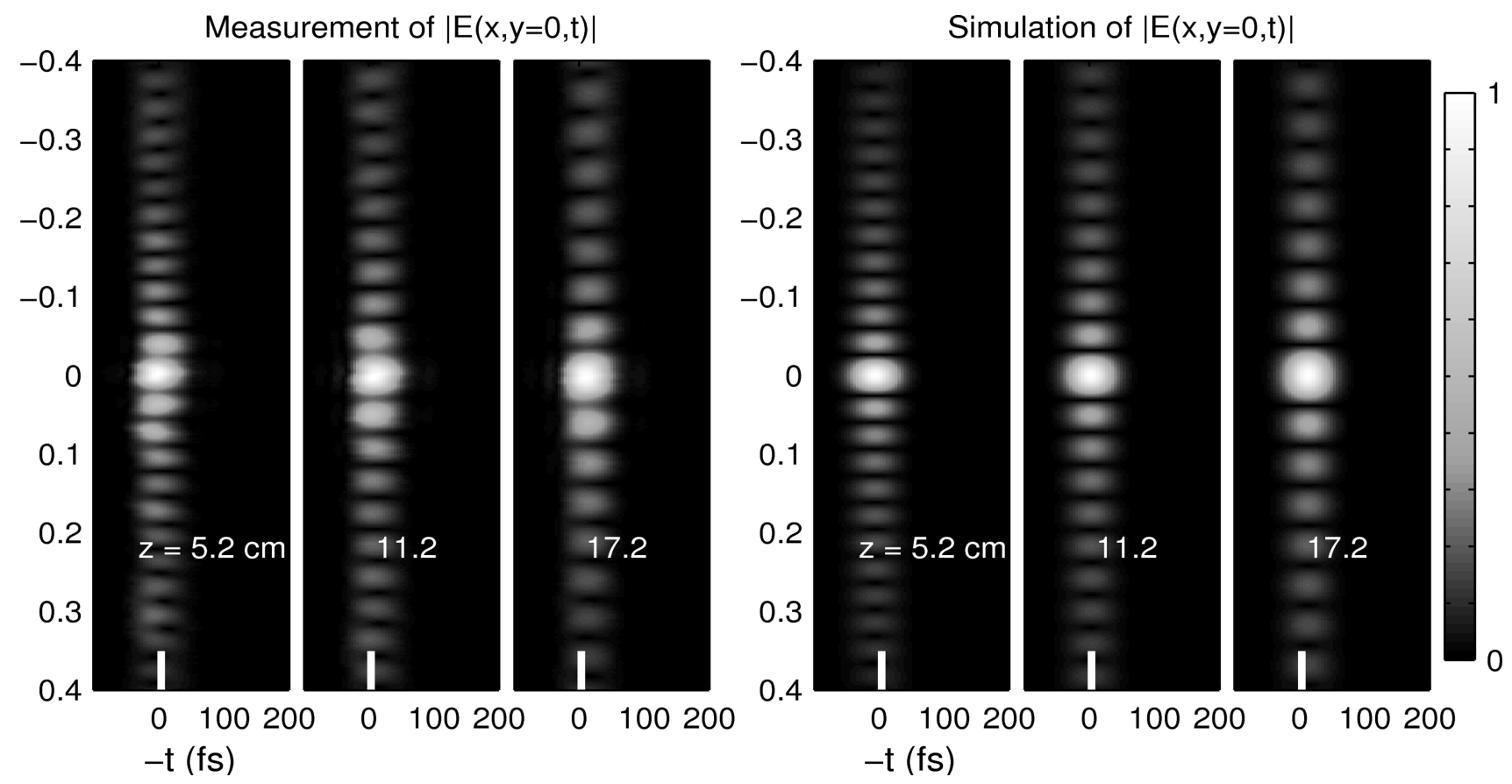

Fig. 2. Comparison of the measured and calculated spatiotemporal profiles of the electric field amplitude of a decelerating Bessel pulse at three positions along the propagation axis $z$.

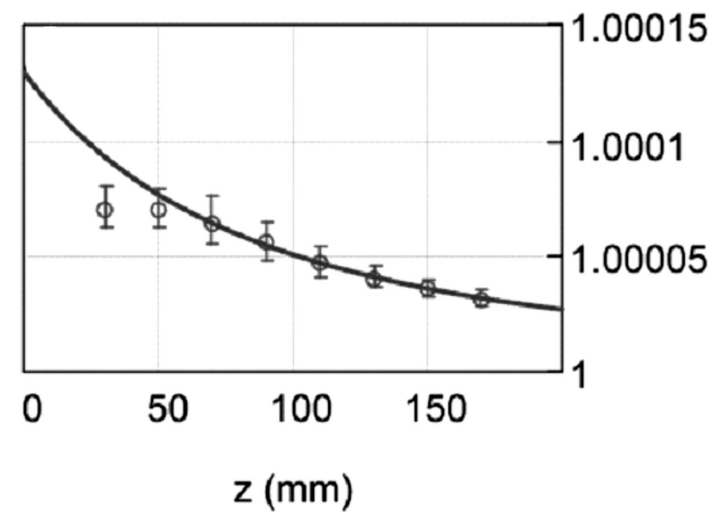

Fig. 3. Experimentally determined group velocity of the decelerating Bessel pulse as a function of the propagation distance. The solid curve shows the theoretical function for comparison.

axis can be considered as the location of the reference pulse if it propagated along the axis $z$ with the Bessel pulses. So, if the Bessel pulse were travelling at the speed of light, then, for each value of $z$, its spatiotemporal intensity would be centred at the same time origin $t=0$ which is emphasized by the white bar in the figure. Again in Fig. 2, note that the superluminal group velocity and the pulse's deceleration are both apparent from the $z$-dependent shifts of the pulses with respect to the origin $t=0$. The time shifts were used for calculation of the pulse's velocity at different propagation distances (see Fig. 3). The decreasing superluminal velocity manifests itself also in the increase of the fringe spacing (increasing radial period of the Bessel profile; see Fig. 2). Accelerating and decelerating Bessel pulses can be also observed when tightly focusing an ultrashort pulse by a lens with spherical aberration [25].

\section{Spatiotemporally recorded diffraction}

Here we consider formation of the Arago spot pulse (for more results on diffraction of pulses through various screens, see the paper by Lõhmus et al., also in this issue).

We propagated ultrashort pulses past an opaque disk of $4 \mathrm{~mm}$ diameter, making a hole in the beam, and we measured the resulting spatiotemporal field at different distances after the aperture to observe its evolution. These measurements reveal the spatiotemporal structure of the weak boundary waves and the brighter spot at the centre of the beam due to their constructive interference, i. e., the spot of Arago, as it is known in conventional diffraction theory for stationary (monochromatic) fields. Interestingly, the plots (like the one in Fig. 4 for a particular propagation distance) reveal that this spot is surrounded by coaxial interference rings and, in the axial region the field, is identical to a decelerating Bessel pulse, which we have considered in the previous section. Moreover, the spot is delayed in time with respect to the main pulse front, and this delay decreases with $z$, indicating a superluminal propagation speed along the $z$ axis (the main pulse front propagates at $c$ ). This occurs, 


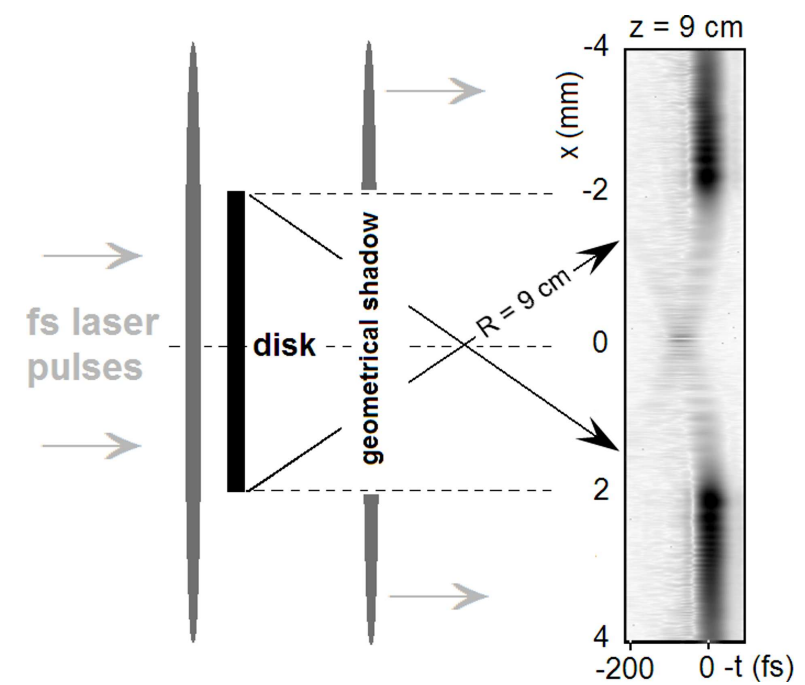

Fig. 4. Schematic of the experiment and the measured time-domain formation of the Arago spot behind an opaque disk with radius $2 \mathrm{~mm}$. Inset: measured electric field amplitude (actually square root of it $-|E|^{1 / 2}-$ for better contrast) $9 \mathrm{~cm}$ behind the disk. This measurement reveals the weak boundary waves that originate from the points along the perimeter of the disk. The boundary waves interfere with the plane wave pulse or the part of the field coming from radii greater than that of the disk, which propagates according to the rules of geometrical optics. Constructive interference between the expanding boundary waves produces a brighter superluminally propagating spot on the axis. The strength of the field is shown "in a negative colormap", in which black corresponds to the maximum strength.

because, as $z$ (or the distance from the disk) increases, the extra distance that the boundary waves must propagate (compared to the main pulse front) to reach the $z$ axis $(x=0)$ decreases, so the relative delay of the boundary waves and the bright spot due to their interference decreases. As a result, the group velocity of the Arago spot - geometrically located at one pole of a luminally expanding spindle torus formed by the boundary diffraction wave pulse - varies from infinity at $z=$ 0 to $c$ for very large values of $z$. Therefore, the spot of Arago is in fact just a decelerating Bessel pulse.

\section{Discussion}

The superluminality of the Bessel-X-type pulses is intriguing. Indeed, while phase velocities greater than $c$ are well known in various fields of physics, a superluminal group velocity is still somewhat taboo, because, at first glance, it seems to be in violation of relativistic causality. However, thanks to the numerous studies throughout the previous century - starting from Sommerfeld's works on the propagation of plane wave pulses in dispersive media - it is well known (see, e. g., a thorough review [30]) that the group velocity need not be a physically profound quantity and by no means should be confused with the signal propagation velocity (which must be less than or equal to $c$ in vacuum). But in the case of Bessel-X-type pulsed waves, no dispersive medium is needed, and still not only is the group velocity superluminal, but the pulse as a whole is also, that is, it rigidly propagates faster than a plane wave.

Naturally, one may feel some unease in accepting this startling circumstance. But here we experimentally observe it in the most direct way. When forced to concede the theoretically and experimentally verified superluminality, one might feel the need to make recourse to statements insisting that the pulse is not a "real" one, but instead simply an interference pattern rebuilt at every point of its propagation axis from truly real plane-wave constituents travelling at a slight tilt with respect to the axis. Such argumentation is not wrong but, alas, leads nowhere. Of course, there is a similarity between the superluminality of the $\mathrm{X}$-wave and a simple geometrical faster-than-light movement of the cutting point in scissors (we refer here to Gedanken experiments described in textbooks on relativity). But in the central highestenergy part of the Bessel-X wave, there is nothing moving at the tilt angle. The phase planes are perpendicular to the axis and move rigidly with the whole pulse along the axis. The Poynting vector, indicating the direction of energy flow, lies also along the axis. However, the energy flux is not superluminal. Hence, to consider the Bessel-X waves as something inferior to "real" waves is not sound. If we thought so, by similar logic we would arrive at the conclusion that femtosecond pulses emitted by a mode-locked laser are not real but "simply an interference" between the continuous-wave laser modes. In other words, one should not ignore the essence of the superposition principle of linear fields, which implies a reversible relation between "resultant" and "constituent" fields and in which no possible orthogonal bases - plane waves or cylindrical (Bessel) waves, for the given example - are inferior to any others.

Another misunderstanding (the author of the review [30] seems to agree) stems from oversight of the fact that there are infinitely many ways to form a pulsed axisymmetric wave packet from single-frequency Bessel beams. They depend on how the radial density of intensity rings in the beam cross-section is related - or whether or not it is related at all - to the beam's temporal frequency. In the case of the Bessel-X pulse, this is a proportionality relation, and therefore the axial group velocity is perfectly defined with a single superluminal value within the whole bandwidth of the wave packet. If, on the contrary, the radial density is 
frequency-independent, we obtain a completely different wave packet which is not a localized wave because it has no definite group velocity over its whole spectrum and therefore spreads as it propagates. But such a wave packet - named the 'pulsed Bessel beam' in the literature - propagates with velocity less than $\mathrm{c}$ and can be used for sending signals along the propagation axis. On the other hand, if one tried to cut a signal "notch" into the core of the Bessel-X pulse, the notch would behave like the 'pulsed Bessel beam' - spreading out while advancing subluminally. This is expected since Maxwell's equations, or the wave equation for EM fields, do not allow superluminal signalling.

\section{Conclusion}

We have performed direct spatiotemporally resolved measurements of pulsed light fields behind various optical refracting and diffracting elements. We believe that time-resolved measurements and a time-domain treatment of diffracting waves not only turn out to be fruitful in modern physical optics, especially in microand meso-optics, but also promote the understanding of diffraction phenomena.

\section{Acknowledgements}

Some support was provided by NSF SBIR grant \#053-9595, and P. B. was also supported by NSF fellowship IGERT-0221600. The other authors were supported by the Estonian Science Foundation, grant \#7870.

\section{References}

[1] J. Durnin, J.J. Miceli Jr, and J.H. Eberly, Phys. Rev. Lett. 58, 1499 (1987)

[2] D. McGloin and K. Dholakia, Contemp. Phys. 46, 15 (2005).

[3] J.N. Brittingham, J. Appl. Phys. 54, 1179 (1983).

[4] R.W. Ziolkowski, Phys. Rev. A 39, 2005 (1988).

[5] J.-Y. Lu and J.F. Greenleaf, IEEE Trans. Ultrason. Ferroelectrics Freq. Control 39, 19 (1992).

[6] I. Besieris, M. Abdel-Rahman, A. Shaarawi, and A. Chatzipetros, Prog. Electromagn. Res. 19, 1 (1998).

[7] J. Salo, J. Fagerholm, A.T. Friberg, and M.M. Salomaa, Phys. Rev. E 62, 4261 (2000).

[8] P. Saari and K. Reivelt, Phys. Rev. E 69, 036612 (2004).
[9] E. Recami and M. Zamboni-Rached, Adv. Imaging Electron Phys. 156, 235 (2009).

[10] E. Gaižauskas, A. Dubietis, V. Kudriašov, V. Sirutkaitis, A. Couairon, D. Faccio, and P. Di Trapani, in: Self-focusing: Past and Present (Springer, 2009), p. 457-479.

[11] Localized Waves: Theory and Applications, eds. H.E. Hernandez-Figueroa, M. Zamboni-Rached, and E. Recami (J. Wiley, New York, 2008).

[12] H. Sõnajalg, M. Rätsep, and P. Saari, Opt. Lett. 22, 310 (1997).

[13] P. Saari and K. Reivelt, Phys. Rev. Lett. 79, 4135 (1997).

[14] K. Reivelt and P. Saari, Phys. Rev. E 66, 056611 (2002).

[15] I. Alexeev, K.Y. Kim, and H.M. Milchberg, Phys. Rev. Lett. 88, 073901 (2002).

[16] R. Grunwald, V. Kebbel, U. Griebner, U. Neumann, A. Kummrow, M. Rini, E.T.J. Nibbering, M. Piché, G. Rousseau, and M. Fortin, Phys. Rev. A 67, 063820 (2003).

[17] F. Bonaretti, D. Faccio, M. Clerici, J. Biegert, and P. Di Trapani, Opt. Express 17, 2276 (2009).

[18] P. Bowlan, R. Trebino, H. Valtna-Lukner, M. Lõhmus, P. Piksarv, and P. Saari, Opt. Lett. 34, 2276 (2009).

[19] H. Valtna-Lukner, P. Bowlan, M. Lõhmus, P. Piksarv, R. Trebino, and P. Saari, Opt. Express 17, 14948 (2009).

[20] M. Clerici, D. Faccio, A. Lotti, E. Rubino, O. Jedrkiewicz, J. Biegert, and P. Di Trapani, Opt. Express 16, 19807 (2008).

[21] J.W. Goodman, Intoduction to Fourier Optics, 3rd ed. (Roberts \& Co, Englewood, 2005).

[22] M. Born and E. Wolf, Principles of Optics, 6th ed. (Pergamon Press, Oxford, 1987).

[23] G.A. Maggi, Ann. di Matem. Pura ed Appl., IIa 16, 21 (1888).

[24] A. Rubinowicz, Nature 180, 160 (1957).

[25] P. Bowlan, U. Fuchs, R. Trebino, and U.D. Zeitner, Opt. Express 16, 13663 (2008).

[26] Z.L. Horváth and Z. Bor, Phys. Rev. E 63, 026601 (2001).

[27] Z.L. Horváth, J. Klebniczki, G. Kurdi, and A. Kovács, Opt. Commun. 239, 243 (2004).

[28] R. Trebino, Frequency-Resolved Optical Gating: The Measurement of Ultrashort Laser Pulses (Kluwer Academic Publishers, Boston, 2002).

[29] P. Bowlan, P. Gabolde, and R. Trebino, Opt. Express 15, 10219 (2007).

[30] P.W. Milonni, J. Phys. B 35, R31 (2002). 


\title{
DIFRAGUOJANTYS IR NEDIFRAGUOJANTYS ŠVIESOS IMPULSAI ERDVĖJE IR LAIKE
}

\author{
P. Saari ${ }^{\text {a }}$, P. Bowlan ${ }^{\text {b }}$,H. Valtna-Lukner ${ }^{\text {a }}$, M. Lõhmus ${ }^{\text {a }}$, P. Piksarv ${ }^{\text {a }}$, R. Trebino ${ }^{\text {b }}$ \\ ${ }^{\text {a }}$ Tartu universiteto fizikos institutas, Tartu, Estija \\ ${ }^{\mathrm{b}}$ Džordžijos technologijos universiteto Fizikos mokykla, Atlanta, JAV
}

\section{Santrauka}

Pateikiame savo naujausių rezultatų apžvalgą apie ultratrumpuju impulsų, perejusių įvairius optinius elementus, evoliuciją. Elektrinis laukas tiesiogiai matuotas erdvejje ir laike metodu, angliškoje literatūroje vadinamu SEA TADPOLE. Mūsų SEA TADPOLE prie- taisas registruoja net $\sim 5 \mu \mathrm{m}$ smulkumo ir vos $\sim 5$ fs trunkančius pokyčius. Eksperimentiniai rezultatai patvirtinti teoriniais skaičiavimais. Aptartas impulsų, turinčių Beselio funkcijos pavidalo radialųji pjūvị, virššviesinis pobūdis. 